\title{
Actively Mode-locked Multiwavelength Fibre Ring Laser Incorporating a Lyot Filter, Hybrid Gain Medium and Birefringence Compensated $\mathrm{LiNbO}_{3}$ Modulator
}

\author{
Colm O'Riordan, Michael J. Connelly, Ivan Evans, Prince M. Anandarajah*, Robert Maher* \\ Liam P. Barry* \\ Optical Communications Research Group, Department of Electronic and Computer Engineering \\ University of Limerick, Limerick, Ireland \\ *RINCE, School of Electronic Engineering, Dublin City University, Dublin, Ireland \\ Tel: (+353) 61202173, e-mail: colm.oriordan@ul.ie
}

\begin{abstract}
A Lyot filter based actively mode-locked multiwavelength fibre ring laser with a hybrid gain medium, consisting of an SOA and EDFA, is presented. Simultaneous mode-locking of up to 8 wavelength channels at $10 \mathrm{GHz}$ is realised using a $\mathrm{LiNbO}_{3}$ based Mach-Zehnder intensity modulator. A wavelength channel spacing of $100 \mathrm{GHz}$ is obtained via appropriate tuning of the Lyot filter length. The effect of modulator birefringence on the performance of the laser spectrum is investigated and a simple method to increase spectral stability is proposed and demonstrated.
\end{abstract}

Keywords: multiwavelength fibre ring laser, mode-locking, Lyot filter, hybrid gain medium, birefringence.

\section{INTRODUCTION}

Multiwavelength lasers have the capacity to replace banks of laser diode transmitters used in dense wavelength division multiplexed (DWDM) transmission systems thereby showing the potential for cost saving. When modelocked they also provide a means to increase the transmission capacity of optical fibre by producing a pulse source that can be exploited by optical time division multiplexing (OTDM) techniques. They also have practical applications in optical gas sensing, optical instrumentation and optical signal processing.

Erbium doped fibre amplifier (EDFA) based multiwavelength fibre ring lasers have been investigated in detail. A major drawback of such systems relates to the homogeneous line broadening of the EDFA gain medium making it difficult to support closely spaced multiple lasing wavelengths at room temperature [1]. A number of techniques have been proposed to increase the number of simultaneous lasing wavelengths that can be supported such as cooling the EDFA to $77 \mathrm{~K}$ [2] or using a frequency shifter in the laser cavity [3]. A more attractive solution is the insertion of a semiconductor optical amplifier (SOA) into the laser cavity to form a hybrid gain medium. The SOA inhomogeneous gain medium serves to suppress the homogeneous line broadening of the EDFA allowing for the generation of multiple closely spaced lasing wavelengths [4]. Furthermore, due to the fast gain saturation profile of the SOA, supermode noise (which is produced when such lasers are harmonically mode-locked) is reduced providing an additional advantage to using this method [5].

There have been several different types of optical filter employed to facilitate the generation of multiwavelength lasing such as Fabry-Perot filters, sampled fibre Bragg gratings, and Mach-Zehnder interferometers. A Lyot filter provides a means for generating a comb filter and is ideally suited to force multiwavelength operation of a fibre ring laser $[6,7]$. The Lyot filter is formed by placing a birefringent medium (a length of polarization maintaining fibre (PMF)) between two polarizers whose axes are aligned $45^{\circ}$ with respect to the polarization axes of the PMF. This provides a solution that exhibits low loss, is fibre compatible and allows for the relatively easy adjustment of the channel spacing by varying the PMF length.

In this paper we present a Lyot filter based Multiwavelength Fibre Ring Laser (MW FRL) using a hybrid gain medium of an SOA and EDFA. The laser is mode-locked at $10 \mathrm{GHz}$ using a LiNbO${ }_{3}$ Mach-Zehnder Modulator (MZM). The wavelength channel spacing is chosen to be $100 \mathrm{GHz}$ via appropriate selection of the Lyot filter length. We also characterize the effect of the modulator birefringence on the performance of the proposed MW FRL in terms of its spectral stability, signal to noise ratio and suppression of the non-optimized wavelength channels. Subsequently, we then go on to show a simple technique to overcome these limitations via the inclusion of a polarizer prior to the modulator in order to compensate for this parasitic birefringence.

The work reported in this paper was supported by Science Foundation Ireland Investigator Grant 02/IN1/I42 and the Irish Research Council for Science, Engineering and Technology (IRCSET). 


\section{EXPERIMENTAL SETUP}

The multiwavelength laser is depicted in Fig. 1. A wavelength channel spacing of $100 \mathrm{GHz}(\sim 0.8 \mathrm{~nm}$ at $1550 \mathrm{~nm}$ ) is achieved by placing $8 \mathrm{~m}$ of PMF between the two polarizers as shown. Channel spacing below $50 \mathrm{GHz}$ is also possible as indicated by our previous work [8]. Isolators ensure unidirectional operation of the laser light. A $20 \mathrm{~nm}$ optical filter is used to remove the gain peak of the EDFA to obtain a more uniform gain spectrum. The two polarization controllers (PC) control the lasing polarization and influence the central lasing wavelength. A portion of the laser output is tapped off using a $3 \mathrm{~dB}$ coupler and sent to an optical spectrum analyzer and a digital communications analyzer for characterization.

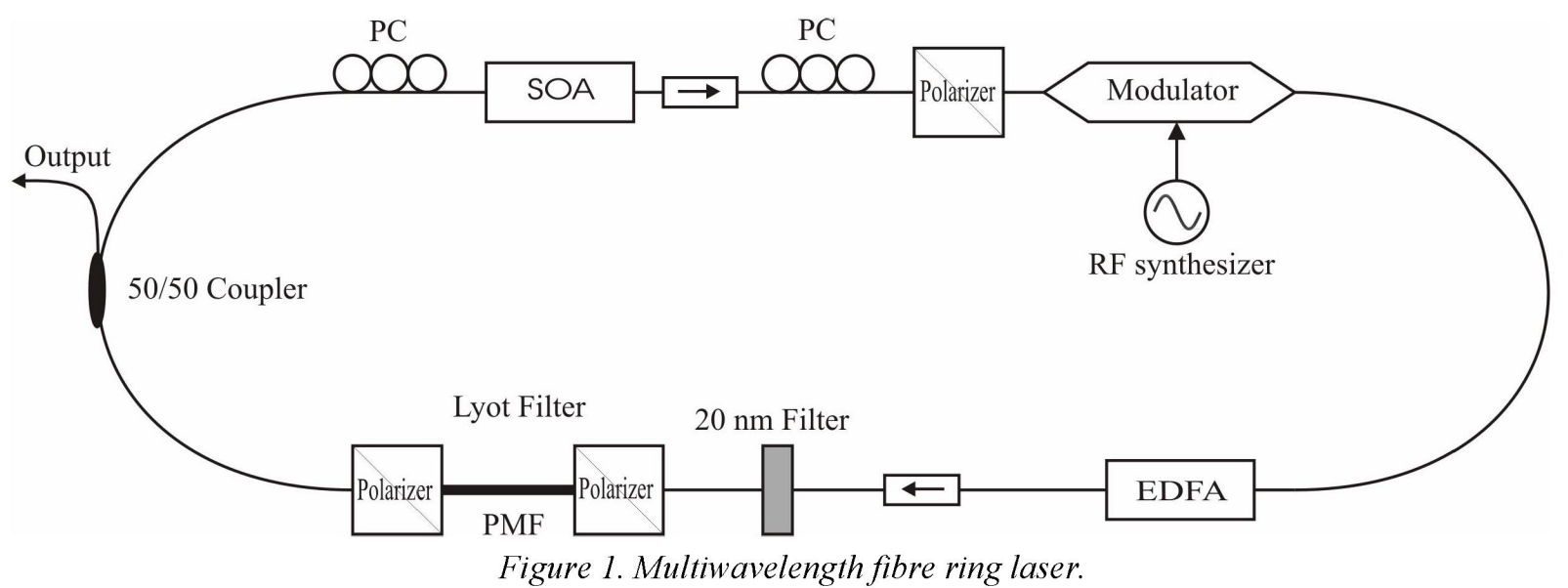

$\mathrm{LiNbO}_{3}$ based Mach-Zehnder intensity modulators are inherently polarization sensitive. This is due to the significant birefringence associated with the substrate material. When used in a configuration similar to that shown in Fig. 1 it can give rise to a parasitic Lyot filter due to the drifting state of polarization (SOP) of the laser light in the single mode fibre (SMF). This drift can be caused by temperature variations and other environmental perturbations. This parasitic filter competes with the Lyot filter and leads to instabilities in the laser spectrum. A typical solution to this problem is to use a polarization controller to compensate for the polarization sensitivity of the modulator. However such a scheme is cumbersome and inefficient. It does not effectively account for the drifting SOP in the laser cavity as the polarization controller has to be adjusted often so that the laser light remains confined to one of the birefringence axes of the modulator. In this work we insert a polarizer, directly before the modulator, whose polarization axis is aligned to one of the birefringence axes of the modulator. This causes the laser light SOP to match that of the polarizer and modulator thereby providing a more efficient and robust defence against laser light SOP drift.

\section{RESULTS}

The laser was actively mode-locked by driving the modulator with an RF sinusoidal waveform at a frequency of $9.729514 \mathrm{GHz}$ which corresponds to approximately the $3088^{\text {th }}$ harmonic of the laser cavity fundamental frequency. The SOA was biased well above transparency to maximise the number of lasing channels that can be supported by the laser and also to increase the supermode noise suppression [8,9]. The EDFA provides supplementary gain and accounts for the loss of the modulator. The polarization controllers, Lyot filter polarizers and modulating frequency were adjusted until the optical spectrum was optimised for a maximum number of lasing channels and maximum uniformity.

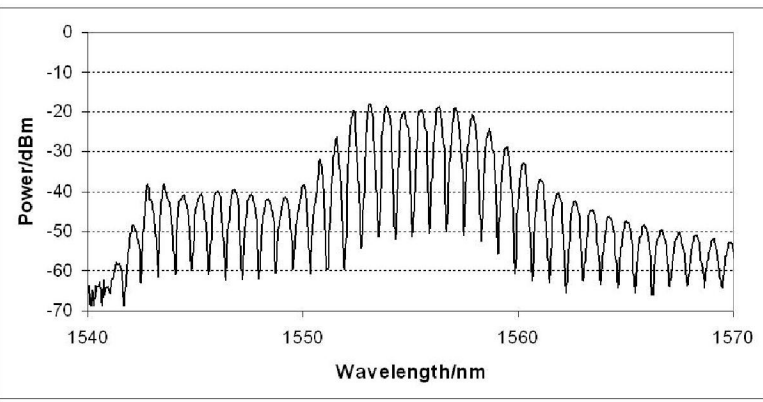

(a)

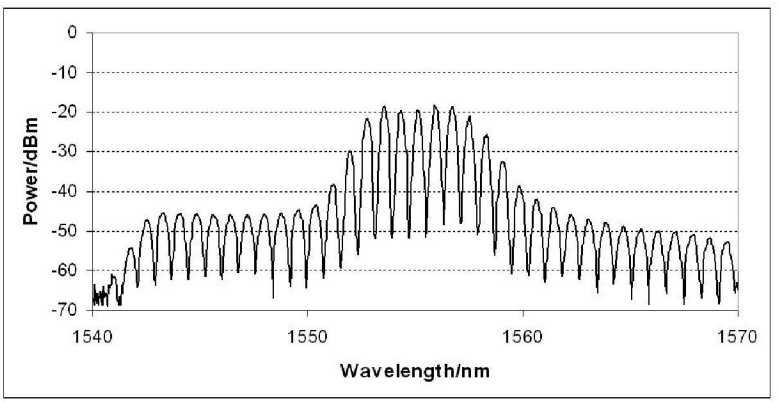

(b)

Figure 2. Multiwavelength mode-locked spectra for (a) Regime A - Using a polarization controller before the modulator and (b) Regime $B$ - after insertion of a polarizer before the modulator. 
The laser's spectral performance was monitored for two regimes. The first regime (Regime A) uses a polarization controller to control the birefringence of the modulator while in the second regime (Regime B) a polarizer is inserted before the modulator whose polarization axis is aligned with one of the birefringence axes of the modulator. Figure $2 a$ shows the optical spectrum for Regime A. Eight lasing channels are observed with a wavelength channel peak standard deviation of $0.9 \mathrm{~dB}$ and a signal-to-noise ratio of approximately $40 \mathrm{~dB}$. The suppression of the non-optimized wavelength channels is $18 \mathrm{~dB}$. Figure $2 b$ shows the optical spectrum for Regime B. In this case seven lasing channels are observed with a wavelength channel peak standard deviation of $1.3 \mathrm{~dB}$. The signal-to-noise ratio is $45 \mathrm{~dB}$ with an improved suppression of the non-optimized wavelength channels of $24 \mathrm{~dB}$. In both cases the wavelength channel spacing is $100 \mathrm{GHz}$ with each individual channel having a $3 \mathrm{~dB}$ bandwidth of approximately $0.25 \mathrm{~nm}$.

There was no significant difference between the pulse trains produced by either regime. The mode-locked pulse train observed on the communications analyzer is shown in Fig. 3. Note that the noise on the pulse train has been removed by oscilloscope averaging. The ringing on the pulses results from the sampling bandwidth limitation of the detector. The measured pulse width was $25 \mathrm{ps}$ which corresponds to the minimum detectable pulse width of the detector. Therefore we assume the actual mode-locked laser pulse width to be less than 25 ps.

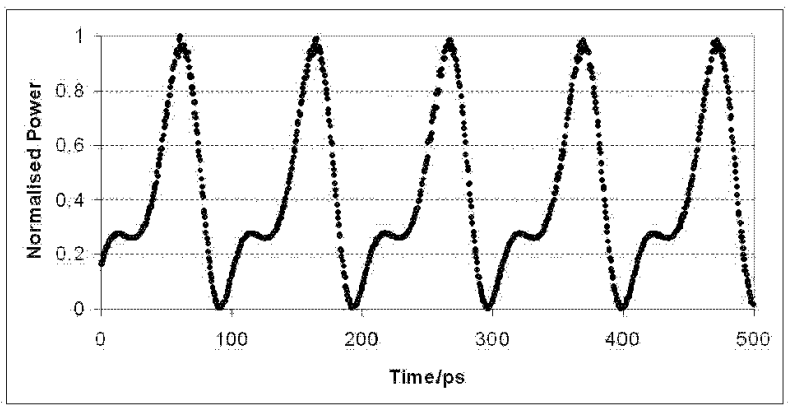

Figure 3. Multiwavelength mode-locked pulse train.

\section{DISCUSSION}

Due to better optimization of the laser cavity SOP, modulating frequency and particularly the Lyot filter polarizers the performance of Regime A marginally exceeds that of Regime B in terms of channel uniformity and number of wavelength channels produced. However we note that with Regime A it is significantly more difficult to tune the laser for optimum spectral performance and to maintain the spectrum in this optimized state. Also with both regimes optimized we note a $6 \mathrm{~dB}$ improvement in the suppression of the non-optimized wavelength channels with the arrangement of Regime $B$.

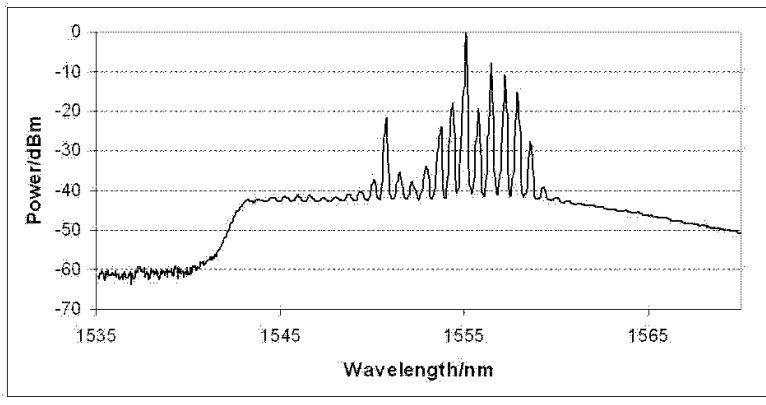

(a)

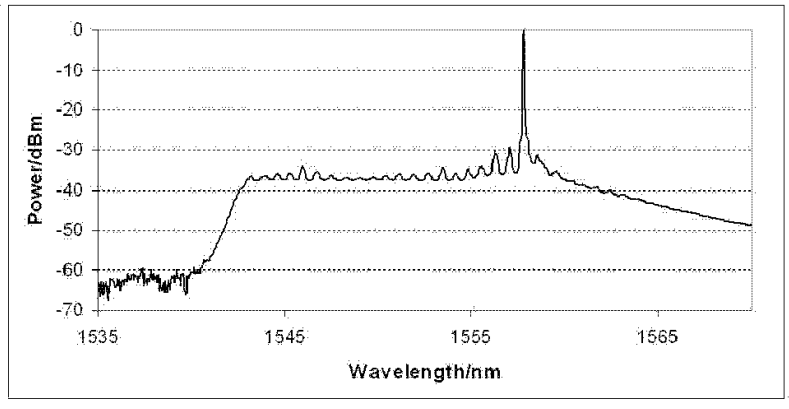

(b)

Figure 4. CW spectra of the laser with the Lyot filter removed for (a) Regime $A$ - using a polarization controller before the modulator and (b) Regime B - inserting a polarizer before the modulator.

These observations can be explained by Fig. 4 which shows the CW laser spectra for both regimes with the Lyot filter removed. Figure $4 a$ shows a significant Lyot filter type response imposed on the CW laser spectrum. This parasitic response is due to the birefringence of the $\mathrm{LiNbO}_{3}$ based modulator. Figure $4 b$ indicates that by inserting a polarizer, whose polarization axis is aligned to one of the modulators birefringence axes, this parasitic filter response is greatly suppressed and the lasing spectrum is essentially single mode as would be expected. The arrangement of Regime B results in a more robust defence against drifting laser light SOP in the cavity thereby ensuring long term stability of the optimised laser spectrum. It also facilitates easier optimization of the laser spectrum and when optimized increases both the suppression of the non-optimised wavelength 
channels and the signal to noise ratio when compared with Regime A. Figure 5 shows the result of drifting laser light SOP while in Regime A. Note the difference between this plot and that of Fig. $2 a$. The parasitic filter response of the modulator impinges on the Lyot filter leading to instability of the laser spectrum. In this case the polarization controller has to be readjusted to optimize the laser spectrum again. This is not an ideal solution as this readjustment can lead to further instability of the laser system due to the high sensitivity of the laser cavity to SOP changes. No such problems are encountered when in Regime B.

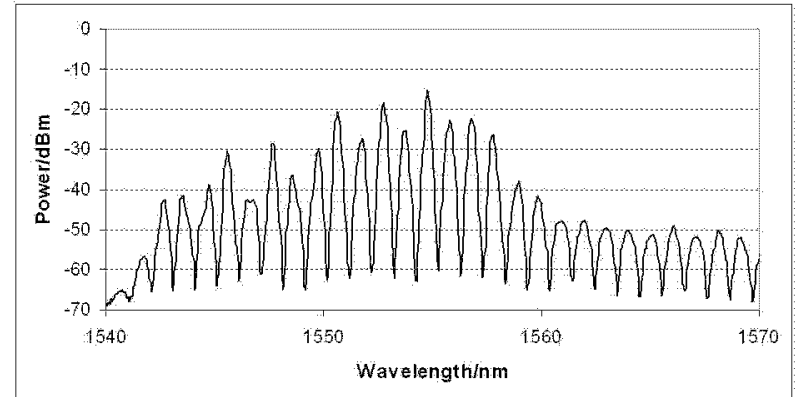

Figure 5. Spectral instability of the multiwavelength mode-locked laser as a result of drifting laser light SOP when in Regime $A$

\section{CONCLUSIONS}

A Lyot filter based multiwavelength fibre ring laser incorporating a hybrid gain medium, consisting of an EDFA and SOA, has been successfully demonstrated. By using an appropriate length of PMF in the Lyot filter a wavelength channel spacing of $100 \mathrm{GHz}$ is realized. Up to 8 wavelength channels are simultaneously modelocked at $10 \mathrm{GHz}$ using a $\mathrm{LiNbO}_{3}$ based Mach-Zehnder intensity modulator. The wavelength channel peaks have a standard deviation of $0.9 \mathrm{~dB}$ indicating excellent uniformity of the multiwavelength spectrum. When using a polarization controller to compensate for the modulator birefringence the suppression of the non-optimized wavelength channels is $18 \mathrm{~dB}$ and the signal to noise ratio is $40 \mathrm{~dB}$. By inserting a polarizer directly before the modulator, whose polarization axis is aligned to one of the modulators birefringence axes, the suppression of the non-optimized wavelength channels improves by $6 \mathrm{~dB}$ to $24 \mathrm{~dB}$ and the signal to noise ratio increases by $5 \mathrm{~dB}$ to $45 \mathrm{~dB}$. Furthermore a significant increase in the long term stability of the laser spectrum is observed.

\section{REFERENCES}

[1] X. Liu, et al., Stable and uniform dual-wavelength erbium-doped fibre laser based on fibre Bragg gratings and photonic crystal fibre, Optics Express, vol. 13, no. 1, pp. 142-147, Jan. 2005.

[2] S. Yamashita and K. Hotate, Multiwavelength erbium-doped fibre laser using intracavity etalon and cooled by liquid nitrogen, Electron. Lett., vol. 32, no. 14, pp. 1298-1299, Jul. 1996.

[3] J.N. Maran, S. LaRochelle, P. Besnard, C-band multi-wavelength frequency-shifted erbium-doped fibre laser, Opt. Comm., vol. 218, pp. 81-86, Jan. 2003.

[4] D.N. Wang, et al., Multiwavelength erbium doped fibre ring laser source with a hybrid gain medium, $O p t$. Comm., vol. 228, pp. 295-301, Sept. 2003.

[5] J. Yao, J. Yao, Z. Deng, Multiwavelength actively mode-locked fibre ring laser with suppressed homogeneous line broadening and reduced supermode noise, Optics Express, vol. 12, no. 19, pp. 4529-4534, Sept. 2004.

[6] R.M. Sova, C.S. Kim, J.U. Kang, Tuneable dual-wavelength all-PM fibre ring laser, IEEE Photon. Technol. Lett., vol. 14, no. 3, pp. 287-289, Mar. 2002.

[7] K. Vlachos, T. Koonen, H. Avramopoulos, 20 channels simultaneous oscillation from a semiconductor fibre laser, in Proc. 15 $5^{\text {th }}$ Ann. IEEE LEOS Conf., Glasgow, paper WZ2, Scotland, 2002.

[8] I. Evans, M.J. Connelly, Multiwavelength mode-locked fibre ring laser with a Lyot filter and a hybrid gain medium, in Proc. OAA, Budapest, Hungary, paper WD6, August 2005.

[9] L. Duan, et al., A stable smoothly wavelength-tunable picosecond pulse generator, IEEE Photon. Technol. Lett., vol. 14, no. 6, pp. 840-842, Jun. 2002. 